\title{
Effective Bandwidth-Based Admission Control for Multiservice CDMA Cellular Networks
}

\author{
Jamie S. Evans and David Everitt
}

\begin{abstract}
In this paper, we develop product form traffic models for single- and multiple-cell code-division multiple-access (CDMA) networks with multiple classes of mobile subscribers. The key feature of this development is the specification of a flexible call admission control procedure that details the numbers of mobiles of each class in each cell that the system operator should allow in order to maintain an acceptable quality of service. Effective bandwidth techniques from the analysis of statistical multiplexing at an asynchronous transfer mode (ATM)based broad-band integrated services digital network (ISDN) link are used to give performance guarantees that overcome the variability in interference levels characteristic of CDMA cellular networks. The result is an admissible region bounded by a finite number of hyperplanes and a simple and efficient call admission policy.

The CDMA mobile network, operating within the admissible region described above, has a very similar form to a circuitswitched network operating with fixed routing. This similarity allows the existing traffic modeling techniques and network management strategies for general loss networks to be applied to CDMA mobile cellular networks. In particular, with standard assumptions on the call arrival processes and holding times, the stationary state distribution has a product form on the truncated state space defined by the call admission strategy.
\end{abstract}

Index Terms - Call admission control, cellular systems, effective bandwidths, multiaccess communication.

\section{INTRODUCTION}

W ITHIN THE last few years, there has been significant interest in digital cellular mobile systems based on code-division multiple access (CDMA) with a standard for the CDMA radio link being developed by Qualcomm [1]-[3].

The majority of the research effort has been concerned with the performance of various modulation and coding schemes in the mobile propagation environment and incorporates important factors such as pseudorandom code design, synchronization, error coding and interleaving, and open- and closed-loop power control algorithms.

However, the network and traffic design issues will be of major significance in any implementation by a cellular network

Manuscript received February 27, 1996; revised October 14, 1997. This work was supported in part by the Australian Telecommunications and Electronics Research Board, the Australian Research Council, and a Telstra Research Laboratories Postgraduate Fellowship. The material in this paper was presented in part at GLOBECOM, Singapore, 1995, and the IEEE Vehicular Technology Conference, Atlanta, GA, 1996.

J. S. Evans was with the Department of Electrical and Electronic Engineering, University of Melbourne, Parkville 3052, Victoria, Australia. He is now with the Department of Electrical Engineering and Computer Science, University of California, Berkeley, CA 94720 USA (e-mail: jse@eecs.berkeley.edu).

D. Everitt is with the Department of Electrical and Electronic Engineering, University of Melbourne, Parkville 3052 Victoria, Australia.

Publisher Item Identifier S 0018-9545(99)00685-4. provider, and to date, this area has been somewhat neglected. It is these network and traffic design issues that we address here. We assume the reader is familiar with the operational features of CDMA cellular systems including power control, soft blocking, soft and softer handoff, diversity, sectorization and exploitation of voice activity cycles [4], [5].

Most capacity analyses of CDMA cellular systems concentrate simply on transmission characteristics and do not consider the real-time operation of the system under a stochastically varying traffic load. Capacity is, therefore, assessed simply by considering a "worst case" scenario of a fixed and equal number of calls in each cell [6], [7]. However, in order to properly design and operate a CDMA cellular system, it is important to be able to quantify the traffic performance of the system, rather than to simply perform a worst case analysis.

The few attempts at traffic modeling of CDMA cellular networks that have appeared in the literature are based on modeling each cell of the network as an independent $M / M / \infty$ queue [8]-[12]. This model corresponds to a system where no calls are blocked and no calls are terminated prematurely. This scenario can be justified to some extent due to the soft blocking property of CDMA systems whereby there is no hard limit on the number of available channels and all users suffer a gradual performance degradation as the load is increased. From a mathematical point of view, the number of users in each cell becomes a Poisson random variable and the total interference can be modeled as a compound Poisson sum. This Poisson stationary distribution for the number of active users in all the above papers employ, and, hence, we may actually replace the negative exponentially distributed holding times by generally distributed random variables.

$M / G / \infty$ models lead to simple techniques for assessing the reverse link traffic capacity of CDMA cellular mobile networks and for investigating its sensitivity to propagation and system parameters. While these models are justifiable based on soft blocking, they are unrealistic from the network operators' viewpoint. At times, when the network becomes heavily loaded, new call requests will be rejected in order to maintain the quality of the existing connections.

Let us assume the network operator is able to accept or reject mobile requests based on the network state which consists of the number of currently active mobiles of each class in each cell of the network. The aim is to only allow network states for which the service quality, as measured by signal to interference ratio, is acceptable with a high probability for every mobile in the network.

An initial scheme for a system with a single user class is as follows. Assume that there are an equal number of active 
mobiles in each cell and maximize this number subject to the quality of service (QoS) constraint. While the calculation of this static capacity is by no means solved for general CDMA mobile networks, there is a body of literature centering on this problem [6]. Once we have this number $N$, our call admission control algorithm simply rejects any call that arrives at a cell with $N$ calls already in progress. We can then model each cell as an independent $M / G / N / N$ loss system and calculate the new call blocking probability from the Erlang B formula.

This approach to resource allocation is similar to fixed channel assignment in conventional channelized systems and does not take advantage of the inherent flexibility of CDMA networks. At times, when neighboring cells are lightly loaded, a cell may be able to hold more than $N$ users and we would like to investigate the possible gains from a more flexible call admission control. Furthermore, the above approach appears to have limited scope for extensions to multiservice networks which will be an integral part of future wireless systems.

A first attempt to model flexible call admission schemes is presented in [13] and [14]. The author uses intercell interference factors to give a set of linear constraints that define the capacity region in terms of the number of users in each cell. These intercell interference factors are constants which are assumed to account for the interference from other cells, however, as the author points out in [15], the other-cell interference demonstrates random behavior strongly dependent on the distribution of mobiles, channel propagation effects, and voice activity. This issue is not addressed in [13] and [14], where the emphasis is on the traffic modeling and performance of the call admission control algorithm using the flexible capacity region.

One possible approach to handle the randomness of the other-cell interference is to use the expected value. If there is much variation in the total interference, however, the QoS may suffer with significant periods of poor radio link quality. On the other hand, the peak interference value would provide a very robust interference factor, but the system would obtain no benefits from statistical multiplexing, a position which is totally untenable for CDMA mobile. We would really like to choose a value lying between the mean and peak interference values which can be used to define a capacity region where a certain QoS constraint will be met.

A similar problem arises in the admission of calls to a broadband integrated services digital network (ISDN) network using asynchronous transfer mode (ATM) where it is useful to assign an effective bandwidth to variable bit rate sources. It is suggested in [15] that the basic concept of effective bandwidth might be usefully applied to cellular CDMA systems, although no details are given. A similar remark appears in [16] where it is mentioned that effective bandwidth concepts may be used in CDMA networks to model the interference from voice speakers in cells at various distances and to incorporate voice and data sources with bursty bit rate requirements. This remark appears to be motivated by the analysis of CDMA networks presented in [8] and [17], although it is not clear that the applications suggested above follow from these references. In [17, Section 9.7], it is shown how an effective bandwidth problem arises in a multiple receiver
CDMA network with multiple classes of user and random resource requirements. This applies only for a macrodiversity model which dismisses the cellular concept and assumes all users are jointly decoded by all receivers in the network. The resulting capacity specification is in terms of the total number of users in the system and leads to an analysis very similar to the single-cell case presented in Section III-A1. We emphasize that this analysis does not apply in the cellular context.

In this paper, we investigate how effective bandwidth concepts can be utilized in the modeling of CDMA cellular mobile networks. These concepts allow us to associate an effective bandwidth to each mobile dependent on its class and location relative to a target cell and to define a capacity region determined by a set of linear constraints. The resultant network model is of similar form to a circuit-switched network and the traffic analysis for the latter can be directly applied to the CDMA cellular model.

We begin in Section II with an examination of the call admission problem for an unbuffered resource shared by multiple classes of user with possibly variable resource requirements. The concept of effective bandwidth is explained and several simple methods for calculating these bandwidths are presented. The important point is that quite general single and multiplecell CDMA cellular networks can be analyzed using the techniques examined in Section II. The appropriate framework for modeling CDMA networks is the subject of Section III which examines the notions of feasibility of network configurations and admissibility of network states. In Section IV, we show how the concepts of Section III apply to a more concrete CDMA network model that operates with two variable rate voice services. Numerical effective bandwidth results for this example network are presented in Section V. In particular, we investigate the dependence of effective bandwidth values on the propagation environment, the system bandwidth and quality of service constraints.

Section VI looks at the traffic modeling of CDMA networks operating within the flexible capacity region developed in the preceding sections and illustrates the similarity to the analysis of fixed circuit-switched networks. While the independence of the cells vanishes in the more dynamic form of operation, the stationary state distribution maintains product form provided some standard assumptions hold for the call arrival processes and holding times. The reduction of many CDMA network models to circuit-switched form is the most important contribution of this paper, the analogy allowing several important operating strategies and analysis techniques from the circuitswitched literature to be directly applied to CDMA mobile systems.

In Section VII, we conclude with a summary of the major insights and results gained in the paper, as well as discussing possible directions for future research.

Before proceeding we note briefly that the developments in this paper concern the capacity of the radio interface only. We do not explicitly deal with capacity limitations due to physical resources and other higher layer factors. However, it is possible to incorporate issues such as modem limitations at base stations into our framework as discussed further in Section VI. 


\section{EFFECTIVE BANDWIDTH CONCEPTS}

The problem of performance not being predictable at call admission time occurs in broad-band ISDN networks using ATM. Calls are accepted to the network according to some call admission policy, but due to the bursty nature of nonvoice traffic, the bursts from different traffic streams may coincide and temporarily require a bit rate higher than the network is able to provide. One popular approach to this problem has been the use of effective bandwidths [16], [18]-[22], [23], where each type of call is assigned an effective bandwidth lying somewhere between the mean rate and the peak rate. An effective bandwidth equal to the peak rate corresponds to a very conservative admission policy which obtains no benefit from the statistical multiplexing of calls, while an effective bandwidth equal to the mean rate corresponds to the expectation that so many calls will be multiplexed together that the variations will be averaged out to produce a nonbursty aggregate traffic stream. The actual values of effective bandwidth assigned will depend on the maximum allowable cell loss rate and on the number of users to be multiplexed together, as well as on the stochastic characteristics of the individual traffic streams.

In this section, we examine a simple problem where the notion of effective bandwidth is useful. The problem arises in the context of controlling the admission of variable bit rate calls to an unbuffered resource. The following section illustrates how a similar problem arises in the admission of users to a CDMA cellular mobile network.

Let $\mathcal{Z}_{+}^{J}$ denote the set of $J$-tuples of nonnegative integers and consider the problem of finding the set of points $\left(N_{1}, \cdots, N_{J}\right) \in \mathcal{Z}_{+}^{J}$ satisfying

$$
P\left(\sum_{j=1}^{J} \sum_{i=1}^{N_{j}} X_{j i}>C\right) \leq \alpha
$$

where $X_{j i}, i=1, \cdots, N_{j}, j=1, \cdots, J$ are independent random variables with distribution function indexed by $j$ and $C$ and $\alpha$ are constants. Identically distributed random variables will be said to belong to the same class and we will call points meeting the constraint of (1) admissible. We note that in the general setting, $X_{j i}$ models the random amount of resource required by call $i$ of class $j, C$ is the total amount of resource available, $\alpha$ is the quality of service requirement, and the $N_{j}, j=1, \cdots, J$ are the number of calls of each class loading the resource.

In what follows we describe two approaches for approximating or bounding the admissible region by the set of points satisfying

$$
\sum_{j=1}^{J} \kappa_{j} N_{j} \leq C
$$

where $\kappa_{j}$ is called the effective bandwidth of the class $j$ random variable.

The first is based on a Gaussian approximation and the second uses the Chernoff bound. The Gaussian approximation is mentioned in [18], where it is not used due to robustness and accuracy problems for large deviations from the mean, and in [19], where it is employed to dimension a collection of variable bit rate sources. The material on the Chernoff bound approach is taken directly from [20, Section 2] and is based on the earlier treatment in [18] which introduces the effective bandwidth concept for unbuffered resources.

\section{A. Gaussian Approximation: Single Class}

For a single class, (1) reduces to $P\left(\sum_{i=1}^{N} X_{i}>C\right) \leq \alpha$. Let $\mu=E\left[X_{i}\right]$ and $\sigma^{2}=\operatorname{var}\left(X_{i}\right)$ for $i=1, \cdots, N$. Then if $\mu<\infty$ and $0<\sigma^{2}<\infty$, the central limit theorem [24] provides justification for approximating the sum of a large number of independent and identically distributed random variables by a Gaussian random variable. Applying this approximation gives the constraint

$$
P(\mathcal{N}(0,1)>(C-N \mu) / \sigma \sqrt{N}) \leq \alpha
$$

where $\mathcal{N}(0,1)$ is a zero-mean unit variance normal random variable. The above constraint is satisfied if and only if $(C-N \mu) / \sigma \sqrt{N} \geq \beta$ where $\beta$ is defined implicitly by

$$
\frac{1}{\sqrt{2 \pi}} \int_{\beta}^{\infty} e^{-t^{2} / 2} d t=\alpha
$$

Continuing on, it is straightforward to show that the constraint can be written in the required form, $\kappa N \leq C$, where the effective bandwidth of a call is given by

$$
\kappa=\mu /\left(1+\frac{2}{z}(1-\sqrt{1+z})\right)
$$

with $z=4 \mu C / \beta^{2} \sigma^{2}$.

\section{B. Gaussian Approximation: Multiple Classes}

From (1), we again invoke the normal approximation and proceeding as in the case of a single class, we are led to the constraint

$$
\sum_{j=1}^{J} N_{j} \mu_{j}+\beta\left(\sum_{j=1}^{J} N_{j} \sigma_{j}^{2}\right)^{1 / 2} \leq C
$$

where $\mu_{j}$ and $\sigma_{j}$ are the mean and variance, respectively, of calls of class $j$ and $\beta$ is as in (3).

Let $\mathcal{R}_{+}^{J}$ denote the positive orthant of $J$ dimensional Euclidean space and call a state $\left(N_{1}, \cdots, N_{J}\right) \in \mathcal{R}_{+}^{J}$ admissible if it satisfies (5). The complement of the admissible region is a convex set in $\mathcal{R}_{+}^{J}$ suggesting the following linear approximations.

1) Homogeneous Gaussian: An overbounding approximation by the hyperplane through the intersection of the admissible region boundary with the $J$ Euclidean axes. The intersection point $\left(0, \cdots, 0, N_{j}^{*}, 0, \cdots, 0\right)$ corresponds to the edge of the single class capacity region for class $j$ calls. We can thus write $N_{j}^{*}=C / \kappa_{j}^{o}$, where $\kappa_{j}^{o}$ is as in (4) with $\mu$ and $\sigma$ replaced by $\mu_{j}$ and $\sigma_{j}$, respectively. It follows that the overbounding linear constraint is given by $\Sigma_{j=1}^{J} \kappa_{j}^{o} N_{j} \leq C$. 
2) Heterogeneous Gaussian: An underbounding approximation by any tangent hyperplane to the boundary of the admissible region. If $\left(N_{1}^{*}, \cdots, N_{J}^{*}\right)$ satisfies (5) with equality, the tangent hyperplane at this point is given by

$$
\sum_{j=1}^{J}\left(N_{j}-N_{j}^{*}\right)\left(\mu_{j}+\frac{\beta \sigma_{j}^{2}}{2 \sigma^{*}}\right)=0
$$

where $\sigma^{*}=\left(\sum_{j=1}^{J} N_{j}^{*} \sigma_{j}^{2}\right)^{1 / 2}$ and the underbounding linear constraint is $\sum_{j=1}^{J} \kappa_{j}^{u} N_{j} \leq C$, where

$$
\kappa_{j}^{u}=\frac{2 C}{2 C-\beta \sigma^{*}}\left(\mu_{j}+\frac{\beta \sigma_{j}^{2}}{2 \sigma^{*}}\right) .
$$

\section{Large Deviation Bound: Single Class}

Consider the single class version of (1) with $M(s)=$ $\log E\left[e^{s X_{1}}\right]$ the common logarithmic moment generating function (LMGF) of the independent random variables. Using the Chernoff bound, the asymptotic behavior of which is covered in Cramer's Theorem [20], [25], it is clear that our original constraint will definitely be satisfied provided

$$
\inf _{s}[N M(s)-s C] \leq \log \alpha .
$$

If equality holds when $N=N^{*}$ and the corresponding infimum is attained with $s=s^{*}$, then our admissible region has the form $\kappa N \leq C$ where the effective bandwidth of a call is given by

$$
\kappa=\frac{C M\left(s^{*}\right)}{C s^{*}+\log \alpha}
$$

\section{Large Deviation Bound: Multiple Classes}

Returning to the multiple class problem with the LMGF of a class $j$ random variable given by $M_{j}(s)$ and once more employing the Chernoff Bound, we know (1) will be satisfied if

$$
\inf _{s}\left[\sum_{j=1}^{J} N_{j} M_{j}(s)-s C\right] \leq \log \alpha .
$$

Call a state $\left(N_{1}, N_{2}, \cdots, N_{J}\right) \in \mathcal{R}_{+}^{J}$ admissible if it satisfies (8), and note the complement of the admissible region is a convex set in $\mathcal{R}_{+}^{J}$ since it is defined as the intersection of $\mathcal{R}_{+}^{J}$ with a collection of half spaces. Similar to the multiple class Gaussian method, we use two linear approximations to the boundary of this admissible region.

1) Homogeneous Chernoff: The hyperplane that overbounds the admissible region is intersecting the points $\left(0, \cdots, 0, N_{j}^{*}, 0, \cdots, 0\right), j=1, \cdots, J$ on the boundary. However, the $N_{j}^{*}$ must be the maximum number of calls allowed on the link when only class $j$ calls are present, and these numbers can be calculated using the single class analysis of the preceding section.
2) Heterogeneous Chernoff: A tangent hyperplane at the point $\left(N_{1}^{*}, N_{2}^{*}, \cdots, N_{J}^{*}\right)$ on the boundary can be used to give a linearization which underbounds the admissible region. If

$$
\inf _{s}\left[\sum_{j=1}^{J} N_{j}^{*} M_{j}(s)-s C\right]
$$

is attained with $s=s^{*}$, then the tail probability will be below $\alpha$ provided

$$
\sum_{j=1}^{J} \kappa_{j}^{u} N_{j} \leq C
$$

where $\kappa_{j}^{u}=C M_{j}\left(s^{*}\right) /\left(C s^{*}+\log \alpha\right)$ is the effective bandwidth of calls of class $j$. This method gives a linear constraint which if satisfied guarantees that the original probabilistic constraint is met.

\section{EFFECTIVE BANDWIDTHS IN CDMA Cellular Networks}

In this section, we investigate how effective bandwidth techniques arise in CDMA cellular networks. Initially, the feasibility of network configurations is investigated. This involves each user achieving acceptable quality of service as measured by reverse link signal to interference ratio. When variability is introduced a probabilistic quality of service condition is required resulting in a similar form to the effective bandwidth problem. We stress our analysis only applies to the reverse link which we assume is orthogonal to the forward link and can thus be treated independently. We concentrate on the reverse link as it is generally accepted that its performance is inferior to that of the forward link [6].

\section{A. Network Configuration Feasibility}

1) Single-Cell Models: Consider a system with bandwidth $W \mathrm{~Hz}$, comprised of a single base station to which $N$ mobiles are connected and suppose that at an arbitrary time instant, mobile $i$ requires a bit rate of $R_{i}$ and a bit energy to interference density ratio of $\left(E_{b} / I_{0}\right)_{i}$. The requirements of mobile $i$ can be encapsulated in the minimum signal to interference density ratio (SIDR) values [6]

$$
\Gamma_{i}=R_{i}\left(E_{b} / I_{0}\right)_{i}, \quad i=1, \cdots, N .
$$

We take the network configuration to consist of the number of mobiles in the system and their corresponding minimum SIDR requirements. A particular network configuration will be called feasible if there exists a set of received power levels that results in every mobile achieving its required SIDR. More specifically the configuration $\left(\Gamma_{1}, \cdots, \Gamma_{N}\right)$ is feasible if there exists nonnegative $P_{1}, \cdots, P_{N}$ which are not all zero and which satisfy

$$
\frac{P_{i}}{\sum_{j=1}^{N} P_{j}} \geq \Gamma_{i} / W, \quad i=1, \cdots, N .
$$

$P_{i}$ is the received power level for mobile $i$, a mobile's own signal is included in the interference it sees, and background 
noise is not modeled (this is a reasonable assumption since the system under consideration is interference limited). It is then straightforward to show that a given configuration is feasible if and only if

$$
\sum_{i=1}^{N} \Gamma_{i} \leq W
$$

2) Multiple-Cell Models: The multicellular networks we study involve each mobile communicating with exactly one base station at any given time. The assignment of a mobile to a base station is determined by factors such as the mobiles location and propagation path losses from the mobile to the prospective base stations (for example, a mobile may connect to the base station offering the best path at any given time). The key point is that we assume base station assignment does not depend on properties (locations, path gains, etc.) of other mobiles.

Alternative approaches include the macrodiversity model of [17, ch. 9], where all users are jointly decoded by all receivers in the network and cellular systems where the single base station assigned to a user may vary with the spatial loading of the network [26]. The feasibility problem in multireceiver or multicellular CDMA networks is intimately related to power control algorithms and we refer the reader to [17] and [26]-[28] for details of more advanced approaches.

For our specified multiple-cell environment, a standard extension of the single-cell analysis would address the following problem: Given the network configuration consisting of the number of mobiles in the system, their required bit rates and bit energy to interference densities, and their propagation gains to every base station in the network, is it possible to assign to each user a receive power level at its fixed target base station in such a way as to satisfy the bit rates and modem requirements of every mobile? If so, then the given network configuration is feasible.

Suppose the network consists of $M$ cells with $N_{m}$ users in cell $m, m=1, \cdots, M$. Let mobile $i$ in cell $m$ have a minimum SIDR requirement in terms of bit rate, $R_{m i}$, and bit energy to interference density ratio, $\left(E_{b} / I_{0}\right)_{m i}$, given by

$$
\Gamma_{m i}=R_{m i}\left(E_{b} / I_{0}\right)_{m i}, \quad i=1, \cdots, N_{m} \quad m=1, \cdots, M .
$$

Furthermore, suppose that mobile $i$ in cell $m$ is received at its target base station with a signal power of $P_{m i}$ and causes interference of power $P_{m i} X_{m i}^{p}$ at the base station of cell $p$ $\left(X_{m i}^{p}\right.$ is thus the ratio of the path gain from mobile $i$ in cell $m$ to the base station of cell $p$ and the path gain from this mobile to its target base station). Then for feasibility of the network configuration, there must exist nonnegative values for the mobiles received powers that satisfy

$$
\frac{P_{m i}}{\sum_{p=1}^{M} \sum_{l=1}^{N_{p}} P_{p l} X_{p l}^{m}} \geq \Gamma_{m i} / W
$$

for $i=1, \cdots, N_{m}$ and $m=1, \cdots, M$. We also require that at least one of the $P_{m i}$ is nonzero so that the above set of inequalities is well defined.
This problem has been studied in the context of narrow-band [29] and wide-band [17, ch. 8] mobile networks and feasibility determined by a constraint on the Perron-Frobenius eigenvalue of the propagation gain matrix.

A much simpler set of constraints which lower bounds the set of feasible network configurations results if we make some assumptions on the received power levels. We assume that received power is directly proportional to minimum SIDR requirement for every user in the system

$$
P_{m i}=\Gamma_{m i}, \quad i=1, \cdots, N_{m} \quad m=1, \cdots, M
$$

where without loss of generality the constant of proportionality is taken to be one. This is a fairly natural constraint that implies the mobile receive power increases as the corresponding SIDR requirement increases. For example, if voice activity monitoring is used, then a user would have a minimum SIDR requirement of zero during silent periods, and it is sensible to give this user a zero receive power level at these times. At a particular base station, the constraint ensures that the SIDR's attained are in the same ratio as the requested SIDR's, moving up and down together as the common interference power varies. The resulting power control algorithm is a simple distributed scheme where each mobile requires only its current SIDR requirement and the path-gain to its target base station to determine its transmit power.

The general feasibility inequalities of (10) become

$$
\sum_{p=1}^{M} \sum_{l=1}^{N_{p}} \Gamma_{p l} X_{p l}^{m} \leq W, \quad m=1, \cdots, M
$$

after application of the power level constraints.

\section{B. Network State Admissibility}

Suppose a user's SIDR requirement comes from one of $J$ classes. The SIDR requirements of calls in the same class have the same deterministic value or are independent and identically distributed random variables. Take the network state to consist of the number of users of each class in each cell of the network with $N_{m j}$ mobiles of class $j$ in cell $m$ for $m=1, \cdots, M, j=1, \cdots, J$. We are interested in the set of network states that lead to network configurations which are feasible, or feasible with high probability. Such network states will be called admissible.

1) Single Cell: Fixed Requirements: If there are $J$ classes of service supported with constant SIDR requirements $\Gamma_{1}, \cdots, \Gamma_{J}$ and $N_{j}$ mobiles of class $j$, then from (9) the network configuration will be feasible and the network state admissible provided

$$
\sum_{j=1}^{J} \Gamma_{j} N_{j} \leq W
$$

This result is equivalent to Lemma 8.1 in [17].

2) Single Cell: Random Requirements: Suppose that the SIDR requirements are independent random variables coming from $J$ classes. If $\Gamma_{j i}$ is the random variable representing the resource requirement of the $i$ th call from class $j$, then the condition on network configuration feasibility applies only to 
a realization of the network configuration and we must use a probabilistic condition for network state admissibility. With $N_{j}$ calls of class $j$, we use

$$
P\left(\sum_{j=1}^{J} \sum_{i=1}^{N_{j}} \Gamma_{j i}>W\right) \leq \alpha .
$$

This is analogous to (1) and can be handled using the multiple class Gaussian approximation or large deviations bound leading to the assignment of an effective bandwidth $\kappa_{j}$ to class $j$ calls in such a way that the network state is admissible if $\sum_{j=1}^{J} \kappa_{j} N_{j} \leq W$. A similar approach can be applied to the macrodiversity model of a multireceiver network as pointed out in [17, ch. 9].

3) Multiple Cells: Fixed Requirements: In the multiplecell environment, there is a great deal of interference variability due to factors such as the randomness in user locations within a cell and shadowing from buildings and land features. Even if the mobiles SIDR requirements are deterministic we must use a probabilistic condition for network state admissibility.

With reference to (11), the admissible region is defined as the set of network states for which

$$
P\left(\sum_{p=1}^{M} \sum_{k=1}^{J} \sum_{l=1}^{N_{p k}} \Gamma_{k} X_{p k l}^{m}>W\right) \leq \alpha, \quad m=1, \cdots, M
$$

where $\Gamma_{k}, k=1, \cdots, J$ are the fixed SIDR requirements and

$$
X_{p k l}^{m} \quad i=1, \cdots, N_{p k}, \quad k=1, \cdots, J \quad p=1, \cdots, M
$$

are independent random variables with distribution indexed by $p$ and $m$. We note that as $m$ varies it does not necessarily index independent sequences. $X_{p k l}^{m}$ models the interference caused at cell site $m$ when mobile $l$ of Type $k$ in cell $p$ is received at its fixed target base station with one unit signal power.

Each of the constraints of (13) can be handled using the effective bandwidth techniques for multiple classes. Whether the Gaussian approximation or large deviations bound is used, the result is an admissible region from the perspective of cell $m$ of the form

$$
\sum_{p=1}^{M} \sum_{k=1}^{J} \kappa_{p k}^{m} N_{p k} \leq W
$$

where $\kappa_{p k}^{m}$ is the effective bandwidth at cell site $m$ of a class $k$ call in cell $p$. By repeating the above constraint at every cell in the network, we arrive at our final admissible region

$$
\mathcal{S}=\left\{\boldsymbol{N} \in \mathcal{Z}_{+}^{M \cdot J} \mid \sum_{p=1}^{M} \sum_{k=1}^{J} \kappa_{p k}^{m} N_{p k} \leq W, m=1, \cdots, M\right\}
$$

or

$$
\mathcal{S}=\left\{N \in \mathcal{Z}_{+}^{M \cdot J} \mid B N \leq W\right\}
$$

where the network state vector $N$ is given by

$$
N=\left(N_{11}, \cdots, N_{1 J}, \cdots, N_{M 1}, \cdots, N_{M J}\right)^{\prime}
$$

and $W$ is an $M$ dimensional column vector with $W$ in every row and $\boldsymbol{B}$ is the $M$ by $M \cdot J$ matrix of effective bandwidth values.

4) Multiple Cells: Random Requirements: If we now allow the SIDR requirements to be random variables, the constraints on network state admissibility of (13) become

$$
P\left(\sum_{p=1}^{M} \sum_{k=1}^{J} \sum_{l=1}^{N_{p k}} \Gamma_{p k l} X_{p k l}^{m}>W\right) \leq \alpha, \quad m=1, \cdots, M
$$

where $\Gamma_{p k l}, l=1, \cdots, N_{p k}, k=1, \cdots, J, p=1, \cdots, M$ are independent random variables with distributions indexed by $k$. Apart from the extra effort involved in dealing with a product of random variables, the results of the previous section apply unchanged leading once more to an admissible region as given in (14).

Before illustrating the above concepts with an example, it is worth noting that the admissible region $\mathcal{S}$ has a form that is very familiar from the study of fixed circuit-switched networks. If we treat each cell as a link with a capacity of one unit, then $\kappa_{p k}^{m}$ is the resource required at link $m$ by a call of class $(p, k)$. The admissible region of (14) consists of all combinations of call classes that can be supported by the links of the network, all of which have bandwidth $W$. This analogy between CDMA networks and circuit-switched networks allows much of the extensive theory developed for the latter to be applied directly to the mobile environment. In particular the traffic modeling of Section VI is taken directly from the literature on loss networks.

\section{A SPECIFIC EXAMPLE: NeTwork MODEL}

In this section, we detail the application of the above concepts to a multicellular CDMA network offering multiple services. Suppose that there are two classes of voice service, the standard service has a data rate of $R_{1} \mathrm{~kb} / \mathrm{s}$ while the premium service operates at $R_{2} \mathrm{~kb} / \mathrm{s}$ with $R_{2}>R_{1}$. We assume that both classes require the same constant $E_{b} / I_{0}$ value and that the system bandwidth is $W \mathrm{MHz}$. Voice activity detection facilities are present at the mobile so that the data rate generated by each mobile can be modeled as an ON/OFF source which we assume has a probability $\psi$ being ON. $\psi$ will be known as the voice activity factor (VAF). Let the resultant ON state SIDR requirements be $\Gamma_{1}$ (standard) and $\Gamma_{2}$ (premium).

Each mobile connects to its closest base station in a uniform, hexagonal, cellular layout and is power controlled to be received at a power proportional to the instantaneous SIDR requirement. Thus, both standard and premium mobiles will not transmit during silent periods and premium users will have twice the standard users receive power level when in the ON state. Path loss is modeled as a deterministic function of distance with no modeling of shadowing or fading. If the transmitter and receiver are separated by $d$ units, then the received power is given by

$$
P_{R}(d)=P_{T} P_{0} d^{-\gamma}
$$




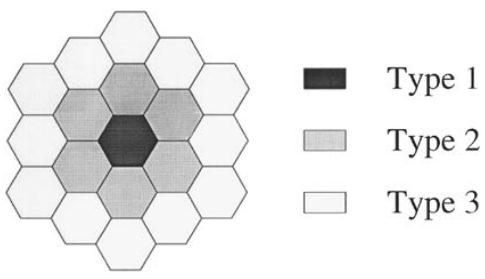

Fig. 1. Types of interference.

where $P_{T}$ is the transmit power and $P_{0}$ and $\gamma$ are independent of distance. $P_{0}$ is a function of carrier frequency, antenna heights, and antenna gains, and we assume it is constant for all paths between mobiles and cell sites. $\gamma$ is the path-loss exponent (PLE) which varies with antenna heights and is typically in the range three to four. The simple model of (16) is accurate for distances from 1 to $20 \mathrm{~km}$ with base station antenna heights greater than $30 \mathrm{~m}$ and in areas with little terrain profile variation [30]. Thus, the model is reasonable for conventional cellular systems in flat service areas but is not accurate in city microcells which employ small cells and low antennas.

Applying (15) leads to the network state admissibility constraints

$$
P\left(\sum_{p=1}^{M} \sum_{k=1}^{2} \sum_{l=1}^{N_{p}} \Gamma_{p k l} X_{p k l}^{m}>W\right) \leq \alpha, \quad m=1, \cdots, M
$$

where $\Gamma_{p k l}, l=1, \cdots, N_{p}, k=1,2, p=1, \cdots, M$ are independent Bernoulli random variables with $k$ indexing the distribution of the standard or premium class and we recall that $X_{p k l}^{m}$ is the interference produced at cell site $m$ when mobile $l$ of class $k$ in cell $p$ is received at its home base station with one unit power. In our simple model of the received power (16), this interference factor is a deterministic function of the distances between mobile $l$ and cell sites $p$ and $m$. However, if we assume the location of the mobile within cell $p$ is a random variable, then the interference factor becomes a random variable. The only barrier to be overcome before the effective bandwidth values can be calculated, is the determination of the distribution functions or moments of these interference factors. Because of symmetry and typically high path-loss exponents in the mobile environment, we need only consider three distributions for the interference factors, as we now show.

Consider the network from the perspective of cell $m$, our target cell. With reference to Fig. 1, the cellular region over which mobiles contribute significant interference to the target base station, is considered in three parts: Type 1 mobiles are located within the target cell, Type 2 mobiles are located in the first layer of surrounding cells, and Type 3 mobiles are located in the second layer of surrounding cells. There are actually two Type 3 cells with slightly different distance from, and orientation to, the target cell, however, we ignore these differences here. Mobiles lying in cells outside the second layer are assumed to contribute a negligible amount to the total other cell interference. This assumption may not be appropriate for low PLE's or situations where the transmit power of certain

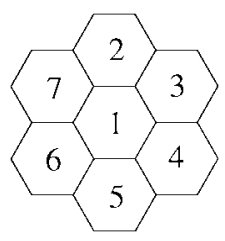

Fig. 2. Seven-cell network of example.

mobiles is high. We simply note here that there is no problem in including more mobile types if required.

Suppose that the locations of mobiles within each cell are independent random variables with a uniform distribution over the cell. By approximating the hexagonal cells with circles, the distribution functions of the interference factors can be obtained in analytic form after expressing the interference as a function of the random user location and using standard transformation techniques [12], [31].

Let $F_{t}(z)$ be the resultant distribution function of a Type $t$ user with $t \in\{1,2,3\}$ and note that $F_{1}(z)=u(z-1)$ where $u(z)$ is the unit step function. Write

$H_{t k}(z)=(1-\psi) u(z)+\psi F_{t}\left(z / \Gamma_{k}\right), \quad t=1,2,3, k=1,2$

and let $n_{t k}^{m}$ be the number of Type $t$ mobiles of class $k$ seen by cell $m$ and take $Z_{t k l}^{m}, l=1, \cdots, n_{t k}^{m}, t=1,2,3, k=1,2$ to be a sequence of independent random variables $Z_{t k l}^{m}$ having distribution function $H_{t k}(z)$. The admissibility conditions of (17) can be rewritten

$$
P\left(\sum_{t=1}^{3} \sum_{k=1}^{2} \sum_{l=1}^{n_{t k}^{m}} Z_{t k l}^{m}>W\right) \leq \alpha, \quad m=1, \cdots, M
$$

and effective bandwidth techniques can be applied to give the linear constraints

$$
\sum_{t=1}^{3} \sum_{k=1}^{2} \kappa_{t k} n_{t k}^{m} \leq W, \quad m=1, \cdots, M
$$

where $\kappa_{t k}$ is the effective bandwidth of a Type $t$ call of class $k$. These inequalities are readily transformed back to the network state domain by expressing the number of calls of each type in terms of the number of calls in each cell. The result is the set of inequalities

$$
\sum_{p=1}^{M} \sum_{k=1}^{2} \kappa_{p k}^{m} N_{p k} \leq W, \quad m=1, \cdots, M
$$

where $\kappa_{p k}^{m}=\kappa_{t k}$ if cell $p$ is a Type $t$ cell with respect to cell $m$.

Suppose the network consists of seven cells as illustrated in Fig. 2. Noting that there are two classes of call, the network state is the 14-dimensional vector

$$
\boldsymbol{N}=\left(N_{1,1}, N_{1,2}, \cdots, N_{7,1}, N_{7,2}\right)^{\prime}
$$

and based on (18) and the simple transformation from the type domain to network state domain, the effective bandwidth matrix $\boldsymbol{B}$ defined in (14) is given by the equation at the bottom of the next page. 
TABLE I

EfFective Bandwidth VAlues $(\mathrm{kHz})$

$(W=1 \mathrm{MHz}, \alpha=0.01$, AND PLE $=4)$

\begin{tabular}{c||c|c|c|c|c}
\hline \multirow{2}{c||}{$\begin{array}{c}\text { Mobile } \\
\text { Type, Class }\end{array}$} & \multicolumn{2}{c|}{ Chernoff Bound } & \multicolumn{2}{c|}{ Gaussian Approximation! } & \\
\cline { 2 - 6 } & Heterogeneous & Homogeneo11s & Heterogeneous & Homogencouls & Mcatl \\
\hline 1,1 & 34.2 & 33.8 & 30.0 & 29.8 & 20.0 \\
\hline 1,2 & 82.4 & 82.4 & 70.4 & 70.2 & 40.0 \\
\hline 2,1 & 2.13 & 2.12 & 1.79 & 1.75 & 1.27 \\
\hline 2,2 & 5.57 & 5.41 & 4.00 & 4.00 & 2.54 \\
\hline 3,1 & 0.0784 & 0.0628 & 0.0729 & 0.0618 & 0.0587 \\
\hline 3,2 & 0.157 & 0.129 & 0.146 & 0.126 & 0.117 \\
\hline
\end{tabular}

TABLE II

EfFective Bandwidth VAlues $(\mathrm{kHz})$

$(W=10 \mathrm{MHz}, \alpha=0.01$, AND PLE $=4)$

\begin{tabular}{|c|c|c|c|c|c|}
\hline \multirow{2}{*}{$\begin{array}{c}\text { Mobile } \\
\text { Type, Class }\end{array}$} & \multicolumn{2}{|c|}{ Chernoff Bound } & \multicolumn{2}{|c|}{ Gaussian Approximation } & \multirow[b]{2}{*}{ Мean } \\
\hline & Heterogeneous & Homogeneous & Heterogeneous & Homogeneols & \\
\hline 1,1 & 23.8 & 23.6 & 22.8 & 22.7 & 20.0 \\
\hline 1,2 & 50.7 & 50.7 & 48.0 & 47.9 & 40.0 \\
\hline 2,1 & 1.50 & 1.48 & 1.42 & 1.40 & 1.27 \\
\hline 2,2 & 3.17 & 3.17 & 2.94 & 2.94 & 2.54 \\
\hline 3,1 & 0.0656 & 0.0601 & 0.0633 & 0.0597 & 0.0587 \\
\hline 3,2 & 0.132 & 0.121 & 0.127 & 0.120 & 0.117 \\
\hline
\end{tabular}

TABLE III

EfFective Bandwidths for Type 1 Standard Mobile $(\mathrm{kHz})(\mathrm{PlE}=4$, Chernoff Bound: Heterogeneous $)$

\begin{tabular}{r||c|c|c|c}
\hline$W(\mathrm{MHz})$ & $\alpha=0.1 \%$ & $\alpha=1.0 \%$ & $\alpha=10 \%$ & Mean \\
\hline \hline 1 & 38.6 & 34.2 & 29.3 & 20.0 \\
\hline 10 & 24.7 & 23.8 & 22.5 & 20.0 \\
\hline
\end{tabular}

Before giving some numerical results we note that the above example is readily extended to include the effects of lognormal shadowing, multiple bursty voice and data services and asymmetric networks [31].

\section{A SPECIFIC EXAMPLE: NumERICAL REsUlts}

Numerical effective bandwidth values are presented in Tables I-III and Figs. 3 and 4.

All values have been normalized to units of kilohertz and are thus appropriate for use in the constraints

$$
\sum_{t=1}^{3} \sum_{k=1}^{2} \kappa_{t k}\left(\gamma, \psi, \alpha, \Gamma_{1}, \Gamma_{2}, W\right), n_{t k}^{m} \leq W \mathrm{kHz}
$$

where $n_{t k}^{m}$ is once more the number of Type $t$ users of class $k$ seen by cell $m$. As shown explicitly in these constraints, the effective bandwidths are functions of the PLE $(\gamma)$, VAF $(\psi)$, quality of service factor $(\alpha)$, ON state SIDR requirements $\left(\Gamma_{1}, \Gamma_{2}\right)$, and system bandwidth $(W)$. The values will also vary with the bound or approximation that is used in their calculation. In all results, we use a VAF of $\psi=0.4$ which corresponds to the expectation that a user is active only $40 \%$

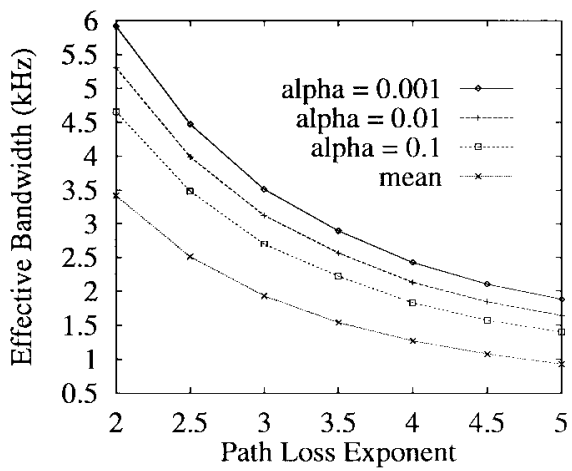

Fig. 3. Effective bandwidths for Type 2 standard mobile ( $W=1 \mathrm{MHz}$, Chernoff bound: heterogeneous).

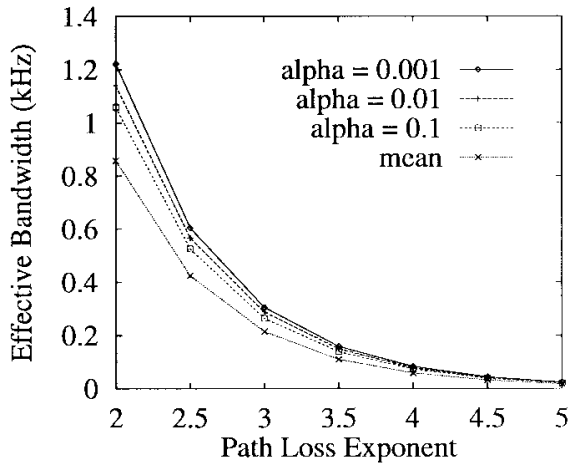

Fig. 4. Effective bandwidths for Type 3 standard mobile $(W=1 \mathrm{MHz}$, Chernoff bound: heterogeneous).

of the time, and we assume $R_{1}=10 \mathrm{~kb} / \mathrm{s}, R_{2}=20 \mathrm{~kb} / \mathrm{s}$ and a common required $E_{b} / I_{0}=7 \mathrm{~dB}$. Thus, $\Gamma_{1}=50 \mathrm{kHz}$ and $\Gamma_{2}=100 \mathrm{kHz}$. When heterogeneous approximations are used the tangent hyperplane touches the admissible region boundary (admissible with respect to target cell $m$ ) at the point

$$
\left(n_{11}^{m}, n_{12}^{m}, n_{21}^{m}, n_{22}^{m}, n_{31}^{m}, n_{32}^{m}\right)=n^{*}(1,1,6,6,12,12)
$$

which corresponds roughly to the expected mix of calls when each cell is offered equal traffic of each class. It is not exactly the expected mix since the higher effective bandwidth class will suffer a higher blocking probability.

In the following results, we show how values vary with PLE's from two to five, quality of service demands of $0.1 \%$, $1 \%$, and $10 \%$, and system bandwidths of 1 and $10 \mathrm{MHz}$

Tables I and II show the six effective bandwidth values for fixed system parameters calculated by the four methods outlined earlier. We note that the Chernoff bound gives more conservative values than the Gaussian approximation and

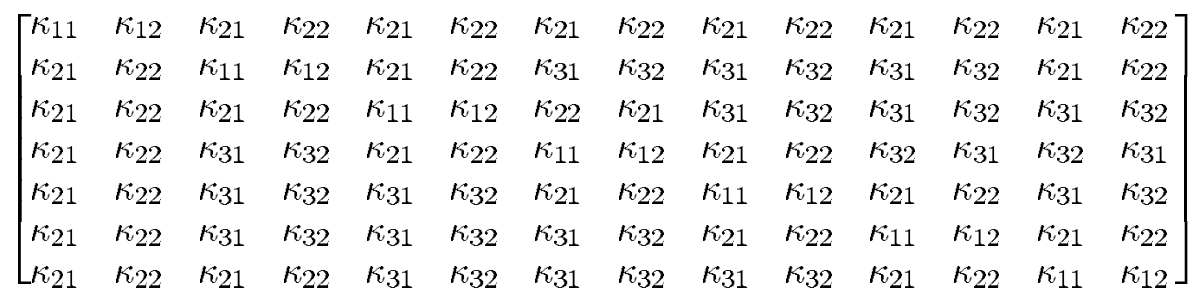


TABLE IV

VALUES OF $n^{*}(\mathrm{PLE}=4)$

\begin{tabular}{|c|c|c|c|c|c|c|c|}
\hline \multicolumn{2}{|c|}{ Parameters } & \multirow[b]{2}{*}{ Simulation } & \multicolumn{2}{|c|}{ Chernoff Bound } & \multicolumn{3}{|c|}{ Guussian Approximation } \\
\hline$W(\mathrm{MHz})$ & $\alpha$ & & Het. & Hom. & Het. & Hon. & $\mathrm{M}[\mathrm{m} \mathrm{d} 1 \mathrm{I}$ \\
\hline 1 & 0.01 & 6 & 6 & 6 & 7 & 7 & 11 \\
\hline 1 & 0.1 & 8 & 7 & 7 & 9 & 9 & 11 \\
\hline 10 & 0.01 & 98 & 95 & 95 & 100 & $10]$ & 117 \\
\hline 10 & 0.1 & 106 & 101 & 101 & 108 & 108 & 117 \\
\hline
\end{tabular}

that there is little difference between the heterogeneous and homogeneous linear approximations. This is an indication that the boundary of the admissible region is close to linear especially for large bandwidth systems. In most cases, values are significantly greater than the corresponding mean although as expected this difference is less pronounced in Table II where the size of the system has been increased relative to Table I and the economy of scale begins to bite. The remaining results use the Chernoff bound with underbounding hyperplane.

Table III shows the predictable variation of $\kappa_{11}$ with $\alpha$ and $W$. Type 1 calls are simply Bernoulli random variables and are essentially independent of PLE except for a very slight variation due to the use of the underbounding linear approximation.

Figs. 3 and 4 show the dramatic influence of PLE on $\kappa_{21}$ and $\kappa_{31}$, respectively, for different quality of service factors. We note that for PLE's greater than 3.5, the contribution of Type 3 calls to the total interference becomes negligible. It is also evident that the Type 3 results cluster closer to the mean than their Type 2 counterparts and it may be reasonable simply to use mean values for Type 3 calls.

To get a feeling for the accuracy of the results obtained from the Chernoff bound and Gaussian approximation we now make some comparisons with results obtained via Monte Carlo simulation. The effective bandwidth values determine our admissible region and it would be useful to compare the resultant admissible regions with the true admissible region (or at least an estimate obtained from simulation). Finding the true admissible region is a computationally intensive task, however, and so instead we will focus on a single point on the boundary of the admissible region. In particular, we calculate the point on the boundary in the direction $(1,1,6,6,12,12)$ by simulation and by all of the methods discussed above.

Let $n^{*}$ be the greatest (integer) value of $n$ such that the state

$$
\left(n_{11}^{m}, n_{12}^{m}, n_{21}^{m}, n_{22}^{m}, n_{31}^{m}, n_{32}^{m}\right)=(n, n, 6, n, 6, n, 12, n, 12, n)
$$

is admissible. For any of the effective bandwidth techniques, we have immediately

$$
n^{*}=W /\left(\kappa_{11}^{m}+\kappa_{12}^{m}+6, \kappa_{21}^{m}+6, \kappa_{22}^{m}+12, \kappa_{31}^{m}+12, \kappa_{32}^{m}\right) .
$$

The resultant values of $n^{*}$ for all of the effective bandwidthbased methods are shown in Table IV for various values of system bandwidth and quality of service factor. Also shown in this table is the corresponding value of $n^{*}$ obtained from Monte Carlo simulation. As expected, the simulated capacity always lies between the value calculated from the Chernoff bound (with underbounding hyperplane) and the capacity predicted based solely on mean values. The Gaussian approximation tends to overestimate capacity, but would generally be more accurate than the Chernoff bound for parameter settings of interest. The advantage of the heterogeneous Chernoff approach is that it is guaranteed to be conservative.

\section{PRoduct Form TrafFic Models}

Up until now we have been concerned with determining a call admission procedure which guarantees that the quality of service constraint (in terms of SIDR requirements) is met. This led to the set of admissible states given in (14). Provided the network state (numbers of mobiles of each class in each cell) lies within the admissible region, then the quality of service constraint will be satisfied. We now turn to the traffic analysis of our CDMA cellular network operating with the specified call admission control algorithm. With standard assumptions on the call arrival and departure processes, our network reduces to a multiservice loss network and the well developed theory for such systems leads directly to expressions for the stationary distribution of the network state and for quantities such as blocking probability.

Consider the state of the network as the stochastic process

$$
N(t)=\left(N_{11}(t), \cdots, N_{1 J}(t), \cdots, N_{M 1}(t), \cdots, N_{M J}(t)\right)^{\prime} .
$$

Assume that calls of class $j$ in cell $m$ are initiated as a Poisson process with rate $\lambda_{m j}$ and as $m$ and $j$ vary over the sets $\{1, \cdots, M\}$ and $\{1, \cdots, J\}$, respectively, they index independent streams. A call request is blocked and cleared from the system if its acceptance would move the state out of the admissible region $\mathcal{S}$ defined in (14). If a call is accepted then it remains in the cell of its origin for a generally distributed holding time with mean $1 / \mu_{m j}$ which is independent of other holding times and of the arrival processes.

With these assumptions, it is well known from the theory of multiservice loss networks [32], [33] that there exists a unique stationary distribution for the stochastic process $N(t)$ with

$$
p(N)= \begin{cases}p_{0} \prod_{m=1}^{M} \prod_{j=1}^{J} \frac{\left(\lambda_{m j} / \mu_{m j}\right)^{N_{m j}}}{N_{m j} !}, & N \in \mathcal{S} \\ 0, & \text { otherwise }\end{cases}
$$

where $p(N)=P(N(t)=N)$ and $p_{0}$ is a normalization constant.

The blocking probability for a class $j$ mobile in cell $m$ is simply

$$
B_{m j}=p_{0} \sum_{\boldsymbol{N} \in \mathcal{S}_{m j}} p(\boldsymbol{N})
$$

where $\mathcal{S}_{m j}$ is the set of states in $\mathcal{S}$ that move out of $\mathcal{S}$ with the addition of one call of class $j$ to cell $\mathrm{m}$. Calculation of the blocking probabilities directly from (20) is infeasible for large networks, however, the product-form solution allows a simple Monte Carlo acceptance-rejection technique to be employed [34], [35]. Alternatively, one can use approximation techniques such as the Erlang fixed point method (or reduced load approximation) from the general theory of loss networks [32], [33], [36].

We mention briefly that the influence of limiting the number of users in any one cell is readily incorporated in the above model by adding extra linear constraints to those in (14). 
This allows the effect of base station hardware limits to be investigated as in [13] and [35]. Numerical results will be presented elsewhere.

\section{CONCLUSIONS}

The key contribution of this paper is the development of robust yet flexible call admission control procedures for multicellular CDMA networks with multiple classes of user and possibly variable bit rate and modem requirements. This development was based on the notion of the feasibility of network configurations and the use of effective bandwidth concepts to handle the variability that characterizes cellular CDMA networks.

The resultant admissible region has a particularly nice form that is very familiar from the analysis of circuit-switched networks. Indeed, a major result of this paper is the demonstration that quite general CDMA mobile networks can be modeled in this circuit-switched form. It is hoped that apart from the traffic modeling above, this analogy will suggest sensible and efficient techniques for the operation and management of CDMA mobile networks. In particular, we note that our models of CDMA networks are the equivalent of circuitswitched networks with fixed routing, equal link capacities, and quite a deal of symmetry. An important observation is that the control of the network operator is essentially limited to call admission control schemes which decide whether an incoming call should be accepted or rejected. Techniques such as trunk reservation may be useful for equalizing blocking probabilities for different call classes, optimizing network revenue, and implementing priority schemes. In any case, the analysis of such techniques as applied to cellular CDMA systems, can draw heavily from the well established theory for their circuitswitched counterparts.

The effective bandwidth models we have presented provide robust capacity specifications for cellular networks operating with fixed base station assignment and simple power control schemes. The major sources of variability modeled are random user locations within a cell and bursty SIDR requirements for each mobile. Results were presented for a deterministic propagation model, however, lognormal shadowing is readily incorporated at the expense of increasing computational overhead.

There is currently a great deal of interest in more general models of cellular networks, particularly those operating with CDMA. Topics include flexible base station assignment schemes, connection to multiple base stations and advanced power control algorithms. The use of such techniques may lead to significant capacity gains over the simple cellular system assumed here. It is also important to assess the performance of the different schemes when factors such as imperfect power control and user mobility are included. These areas will be the subject of future work.

\section{ACKNOWLEDGMENT}

The authors would like to thank the anonymous reviewers for their comments and suggestions which helped to improve the presentation of the paper.

\section{REFERENCES}

[1] A. Salmasi, "Advanced wireless telecommunications systems employing code division multiple access," in Proc. IEEE Int. Symp. Spread Spectrum Techniques and Applications, London, U.K., Sept. 1990.

[2] A. Salmasi and K. S. Gilhousen, "On the system design aspects of code division multiple access (CDMA) applied to digital cellular and personal communications networks," in Proc. IEEE Veh. Technol. Conf., St. Louis, MO, May 1991, pp. 57-62.

[3] R. Padovani, "Reverse link performance of IS-95 based cellular systems," IEEE Personal Commun., vol. 1, no. 3, pp. 28-34, 1994.

[4] W. C. Y. Lee, "Overview of cellular CDMA," IEEE Trans. Veh. Technol., vol. 40, pp. 291-302, May 1991.

[5] R. Kohno, R. Meidan, and L. B. Milstein, "Spread spectrum access methods for wireless communications," IEEE Commun. Mag., vol. 33, pp. 58-67, Jan. 1995.

[6] K. S. Gilhousen, I. M. Jacobs, R. Padovani, A. J. Viterbi, L. A. Weaver, and C. E. Wheatley, "On the capacity of a cellular CDMA system," IEEE Trans. Veh. Technol., vol. 40, pp. 303-312, May 1991.

[7] R. L. Pickholtz, L. B. Milstein, and D. L. Schilling, "Spread spectrum for mobile communications," IEEE Trans. Veh. Technol., vol. 40, pp. 313-322, May 1991.

[8] A. M. Viterbi and A. J. Viterbi, "Erlang capacity of a power controlled CDMA system," IEEE J. Select. Areas Commun., vol. 11, pp. 892-900, Aug. 1993.

[9] A. O. Fapojuwo, "Radio capacity of direct sequence code division multiple access mobile radio systems," Proc. Inst. Elect. Eng., vol. 140, pp. 402-408, Oct. 1993.

[10] M. Frullone, G. Riva, P. Grazioso, and M. Missiroli, "Comparisons of multiple access schemes for personal communication systems in a mixed cellular environment," IEEE Trans. Veh. Technol., vol. 43, pp. 99-109, Feb. 1994.

[11] W. Lavery and D. Everitt, "Analysis of the uplink teletraffic behavior in CDMA cellular systems," in Proc. IEEE Veh. Technol. Conf., Stockholm, Sweden, June 1994, pp. 868-872.

[12] J. S. Evans and D. Everitt, "On the teletraffic capacity of CDMA cellular networks," IEEE Trans. Veh. Technol., vol. 48, pp. 153-165, Jan. 1999.

[13] D. Everitt, "Analytic traffic models of CDMA cellular networks," in Proc. Int. Teletraffic Congress, Antibes Juan-les-Pins, France, June 1994, pp. 349-356.

[14] networks," Proc. IEEE, vol. 82, pp. 1371-1382, Sept. 1994.

[15] _ _ "Variability of traffic performance in CDMA cellular networks," in Proc. Int. Workshop Multi-Dimensional Mobile Communications, Niigata, Japan, Nov. 1994.

[16] D. Mitra and J. A. Morrison, "Erlang capacity and uniform approximations for shared unbuffered resources," IEEE/ACM Trans. Networking, vol. 2, pp. 558-570, Dec. 1994.

[17] S. V. Hanly, "Information capacity of radio networks," Ph.D. dissertation, Univ. Cambridge, Cambridge, U.K., Aug. 1995.

[18] J. Y. Hui, "Resource allocation for broadband networks," IEEE J. Select. Areas Commun., vol. 6, pp. 1598-1608, Dec. 1988.

[19] R. Guérin, H. Ahmadi, and M. Naghshineh, "Equivalent capacity and its application to bandwidth allocation in high-speed networks," IEEE J. Select. Areas Commun., vol. 9, pp. 968-981, Sept. 1991.

[20] F. P. Kelly, "Effective bandwidths at multi-class queues," Queueing Syst., vol. 9, pp. 5-16, 1991.

[21] A. I. Elwalid and D. Mitra, "Effective bandwidth of general Markovian traffic sources and admission control of high speed networks," IEEE/ACM Trans. Networking, vol. 1, pp. 329-343, June 1993.

[22] G. Kesidis, J. Walrand, and C. Chang, "Effective bandwidths for multiclass Markov fluids and other ATM sources," IEEE/ACM Trans. Networking, vol. 1, pp. 424-428, Aug. 1993.

[23] R. Griffiths and P. Key, "Adaptive call admission control in ATM networks," in Proc. Int. Teletraffic Congress, Antibes Juan-les-Pins, France, June 1994, pp. 1089-1098.

[24] P. Billingsley, Probability and Measure, 2nd ed. New York: Wiley, 1986.

[25] J. A. Bucklew, Large Deviations Techniques in Decision, Simulation, and Estimation. New York: Wiley, 1990.

[26] S. V. Hanly, "An algorithm for combined cell-site selection and power control to maximize cellular spread spectrum capacity," IEEE J. Select. Areas Commun., vol. 13, pp. 1332-1340, Sept. 1995.

[27] D. Mitra and J. A. Morrison, "A distributed power control algorithm for bursty transmissions on cellular, spread spectrum wireless networks," in Proc. WINLAB Workshop on 3rd Generation Wireless Information Networks, East Brunswick, NJ, Apr. 1995, pp. 275-285. 
[28] R. D. Yates, "A framework for uplink power control in cellular radio systems," IEEE J. Select. Areas Commun., vol. 13, pp. 1341-1347, Sept. 1995.

[29] G. J. Foschini and Z. Miljanic, "A simple distributed autonomous power control algorithm and its convergence," IEEE Trans. Veh. Technol., vol. 42, pp. 641-646, Nov. 1993.

[30] M. Hata, "Empirical formula for propagation loss in land mobile radio services," IEEE Trans. Veh. Technol., vol. 29, pp. 317-325, Aug. 1980.

[31] J. S. Evans, "Traffic modeling of cellular mobile communications systems operating with code division multiple access," M.S. thesis, Univ. Melbourne, Australia, July 1995.

[32] F. P. Kelly, "Loss networks," Ann. Appl. Prob., vol. 1, no. 3, pp. 317-378, 1991

[33] K. W. Ross, Multiservice Loss Models for Broadband Telecommunication Networks. New York: Springer-Verlag, 1995.

[34] D. Everitt and N. W. Macfadyen, "Analysis of multicellular mobile radiotelephone systems with loss," Brit. Telecom Technol. J., vol. 1, no. 2, pp. 37-45, Oct. 1983.

[35] D. Everitt and D. Manfield, "Performance analysis of cellular communications systems with dynamic channel assignment," IEEE J. Select. Areas Commun., vol. 7, pp. 1172-1180, Oct. 1989.

[36] F. P. Kelly, "Blocking probabilities in large circuit-switched networks," Advances in Applied Probability, vol. 18, pp. 473-505, 1986.

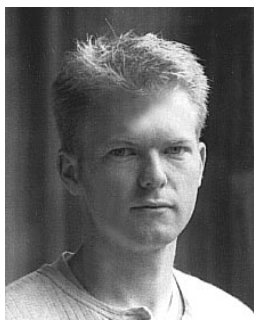

Jamie S. Evans received the B.Sc. degree in physics and the B.E. degree in computer engineering from the University of Newcastle, Australia, in 1992 and 1993, respectively, and the M.Eng.Sc. and Ph.D degrees in electrical engineering from the University of Melbourne, Australia, in 1995 and 1998, respectively.

He is currently a Post-Doctoral Fellow in the Department of Electrical Engineering and Computer Science, University of California, Berkeley. His interests are in stochastic filtering and control theory and in the design and analysis of broad-band and wireless communications networks.

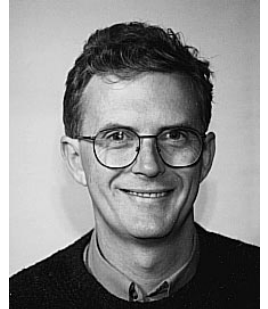

David Everitt received the B.E. and Ph.D. degrees in electrical engineering from the University of Queensland, Australia, in 1976 and 1981, respectively.

From 1981 to 1986, he was with British Telecom Research Laboratories. From 1987 to 1988, he was with Telecom Australia Research Laboratories. Since March 1989, he has been with the Department of Electrical and Electronic Engineering, University of Melbourne, Australia. His interests are in performance engineering for communication networks, especially cellular mobile communications networks, local-area networks, and all-optical networks. 